\title{
Aspirin for strokes and transient ischaemic attacks
}

\author{
No panacea
}

Despite Samuel Wesley's statement over 200 years ago that "there cannot be an absolute panacea-a medicine that will cure every disease incident to the human body," the ancient medicine aspirin is beginning to be perceived as the panacea for the diseases of the twentieth century. Its efficacy in treating conditions ranging from fever to arthritis has long been known, but now it is recognised as effective in the secondary prevention of strokes and heart attacks ${ }^{1} ; 300 \mathrm{mg}$ daily taken for a few years after a transient ischaemic attack or minor ischaemic stroke reduces non-fatal strokes or myocardial infarctions by about a third and deaths from vascular disease by about a sixth. Aspirin is also being tested in conditions as divergent as diabetes mellitus and preeclampsia.

Three problems suggest, however, that this effective, cheap, and simple treatment may not be a panacea for cerebrovascular disease: the need to exclude intracerebral haemorrhage before starting treatment; the temptation to prescribe aspirin and not consider alternative diagnoses or additional treatments; and the widespread use of low doses whose efficacy is uncertain.

The most reliable way to exclude intracerebral haemorrhage in a patient with a stroke is computed tomography within two weeks after the onset of the stroke. ${ }^{2}$ Yet only two fifths of health districts in England and Wales have such a scanner. ${ }^{3}$ Thus excluding cerebral haemorrhage is difficult in Britain because of the lack of facilities for computed tomography. (This is in striking contrast to much of Europe and North America, where computed tomography is widely available.) Inevitably, doctors who do not have ready access to a computed tomography scanner may start giving aspirin to patients with strokes without first performing computed tomography; such a policy will lead to some patients with primary intracerebral haemorrhage being wrongly treated with aspirin.

We have no information on the risks of treating patients who have had a primary intracerebral haemorrhage with aspirin, and such information is never likely to become available as it would be unethical to perform the trial. Aspirin may also increase the risk of subsequent intracranial haemorrhage in patients who at the start of treatment have no history of intracranial haemorrhage. The scanty data from trials in people without vascular disease ${ }^{4}$ and in patients with transient ischaemic attacks or minor ischaemic strokes ${ }^{5}$ are compatible both with aspirin doubling the risk of intracranial bleeding and with there being no excess risk at all. Further data are being collected. Although there are no data on the effects of treating patients with proved intracranial haemorrhage with aspirin, it seems prudent to avoid treating them. Thus until facilities for computed tomography are more widely available in Britain doctors will have to balance the risk of not doing a scan and treating the patient blindly with aspirin (thus possibly exacerbating an undiagnosed intracerebral haemorrhage) or not giving aspirin (and possibly denying the patient a useful treatment).

The second problem is that prescribing aspirin may signal a premature end to the search for the correct diagnosis. Doctors may be tempted to simply prescribe aspirin rather than investigate the patient to exclude treatable conditions that may mimic stroke and transient ischaemic attacks-focal epilepsy, migraine, hypoglycaemia, and cardiac arrhythmias, to name but a few. For example, a patient with dizzy spells may be misdiagnosed as having transient ischaemic attacks and be treated with aspirin until an electrocardiograph shows episodic complete heart block and indicates that treatment should be cardiac pacing rather than aspirin. Even if patients' transient ischaemic attacks have been correctly diagnosed treatable risk factors such as hypertension, hyperlipidaemia, and smoking may not be rigorously dealt with "because the patient is being treated" (with aspirin). Doctors may not think it worth while to refer patients for treatments such as carotid endarterectomy once treatment with aspirin has been started.

Yet aspirin reduced non-fatal stroke or myocardial infarction by "only" a third so there is plenty of scope for other measures. Data from overviews of randomised trials suggest that reducing diastolic blood pressure by $5-10 \mathrm{~mm} \mathrm{Hg}$ over a few years in patients who have had a transient ischaemic attack might reduce subsequent strokes by $40-50 \%$; and reducing plasma cholesterol concentration by $10 \%$ over a few years might reduce subsequent events from coronary heart disease by a fifth or more.

The third problem is the correct dose of aspirin. Though some laboratory evidence supports using very low doses of aspirin (less than $30 \mathrm{mg}$ daily), ${ }^{8}$ the smallest dose proved to work in clinical trials of long term secondary prevention of stroke and heart attack is $300 \mathrm{mg}$ daily.' Smaller doses are being tested in at least two trials in patients who have had transient ischaemic attacks - the Dutch transient ischaemic attack trial ${ }^{8}$ (30 mg) and the Swedish low dose aspirin trial (30 
$\mathrm{mg})$. A recent trial of short term use in patients who have had acute myocardial infarctions has shown that $160 \mathrm{mg}$ aspirin daily reduces mortality after five weeks by a fifth. ${ }^{9}$ Despite the lack of evidence from clinical trials many patients with transient ischaemic attack and stroke are treated with $75 \mathrm{mg}$ daily (a dose enshrined in the current British National Formulary), with the risk that they may be receiving an inadequate dose, albeit a safe one. On the basis of properly conducted clinical trials patients with transient ischaemic attacks and minor ischaemic strokes and no previous problems of peptic ulceration should be treated with $300 \mathrm{mg}$ aspirin daily. The dose may be reduced to $150 \mathrm{mg}$ and then $75 \mathrm{mg}$ if gastrotoxicity is a problem since gastrotoxicity is related to dose. ${ }^{+}$

Aspirin is therefore not a panacea for every patient with a suspected stroke or an undiagnosed "funny turn." But if used in the correct dose in patients with definite transient ischaemic attacks or ischaemic stroke proved by computed tomography it is one of the several effective forms of secondary prevention.

Senior Lecturer,

PETER SANDERCOCK

Department of Clinical Neurosciences,

Western General Hospital,

Edinburgh EH4 2XU
1 Antiplatelet Trialists' Collaboration. Secondary prevention of vascular disease by prolonged antiplatelet treatment. Br Med f 1988;296:320-31.

2 Dennis MS, Bamford JM, Molyneaux A, Warlow CP. Rapid resolution of primary intracerebral haematoma on computed tomograms of the brain. BrMed f 1987;295:379-81.

3 King's Fund Forum. Consensus conference: treatment of stroke. Br Med f 1988;297:126-9.

4 Steering Committee of the Physicians' Health Study Research Group. Preliminary report: finding from the aspirin component of the ongoing Physicians Health Study. $N$ Engl $q$ Med 1988;318:262-4.

5 UK-TIA Study Group. United Kingdom transient ischaemic attack (UK-TIA) trial: interim results. Br Med f 1988;296:316-20.
6 McMahon S, Culter JA, Furberg CD, Payne GH. The effects of drug treatment for hypertension on morbidity and mortality from cardiovascular disease: a review of the randomised controlled trials. Prog Cardiovasc Dis 1986;29(suppl 1):99-118.

7 Peto R, Yusuf S, Collins R. Cholesterol lowering trial results in their epidemiologic context. Circulation 1985;72(III):451.

8 The Dutch TIA Study Group. The Dutch TIA trial: protective effects of low-dose aspirin and atenolol in patients with TIA or non-disabling stroke. Stroke 1988;19:512-7.

ISIS-2 (second international study of infarct survival) Collaborative Group. Randomised trial of intravenous streptokinase, oral aspirin, both, or neither in 17189 cases of suspected acute myocardial infarction: ISIS-2. Lancet 1988;ii:349-60.

\section{Acute failure of artificial heart valves}

\section{The risk is small}

The recent withdrawal from the market of a mechanical heart valve has once again focused attention on the imperfect nature of substitutes for heart valves. ${ }^{1}$ Reassurance can and should be given, however, to patients with heart valves that such acute failures are exceedingly rate.

Over 5000 artificial heart valves are implanted each year in Britain. Though the incidence of rheumatic fever and chronic rheumatic valvular disease has decreased substantially, overall numbers of valve replacements have been maintained by the increasing incidence of degenerative valve disease in an aging population. The change in the type of diseases in the valves combined with awareness of the imperfections of artificial heart valves have encouraged cardiac surgeons to carry out whenever possible conservative operations that avoid implanting artificial valves. The impressive work of Carpentier et al in France and Cosgrove et al in the United States has shown what can be achieved with conservative operations. ${ }^{2}{ }^{3}$ In Britain, however, only about $12 \%$ of operations on mitral valves and $1-2 \%$ of those on aortic valves are conservative. Conservative operations are favoured for the tricuspid valve, but few such procedures are performed.

Most British patients thus have a diseased valve replaced by an artificial valve-either a mechanical valve (manufactured from totally synthetic materials) or a tissue or bioprosthetic valve (using biological tissue). In 1986 over half (54\%) of all artificial valves implanted in Britain were mechanical. In 1987 this figure rose to almost two thirds (63\%).

The operative mortality of valve replacements has fallen steadily over the past decade. Overall it is now around $5 \%,{ }^{4}$ and even when two or three valves are replaced at the same operation the operative mortality is only $8-11 \%$. Medium and long term mortality and morbidity reflect both the intrinsic imperfections of artificial valves and the inevitable pathological complications associated with implanted artificial valves (infective endocarditis, thromboembolism, and haemorrhage related to anticoagulants) that are not primarily associated with dysfunction of the valve mechanism. Endocarditis, thromboembolism, and haemorrhage related to anticoagulants each occur about one to four times for each 100 patient years of follow up. ${ }^{5}$ These incidences are accepted as the non-preventable consequences of implanting artificial valves.

By contrast, acute failure of the mechanism of an artificial valve is rare, particularly for mechanical valves made from totally synthetic materials. Tissue of bioprosthetic heart valves undergo progressive tissue degeneration rather than sudden failure. The most recent valve failures have been with the Edwards-Duromedics bileaflet valve and the Medtronic Hall single leaflet valve. The Duromedics valve fails because a leaflet escapes whereas in the Medtronic valve the leaflet fractures. Embolisation of the leaflet or part of the leaflet may occur and severe valve regurgitation immediately develops. The sequence is similar to that seen in the acute failure of Bjork-Shiley disc valves reported in 1983, in which fracture of one of the metal struts in the valve ring allowed the valve leaflet to escape.

The Duromedics valve fails 0.04 times in every 100 patient years, whereas the Medtronic valve has failed only three times, although 30000 have been implanted. Patients are thus up to 100 times more likely to develop infective endocarditis, thromboembolism, and haemorrhage related to anticoagulants than to sustain acute mechanical failure of the valve. These data should not, however, encourage complacency. Acute failure of a mechanical valve carries a high immediate mortality; only about a third of patients have survived a strut fracture of the Bjork-Shiley valve. ${ }^{6}$ So far half of the patients have survived an escape of the Duromedics valve leaflet and two of the three patients survived acute failure of the Medtronic valve.

The chances of survival are increased by clinical awareness, rapid diagnosis, and immediate referral for reoperation. The diagnosis of acute failure of the mechanical valve should be suspected in any patient known to have a mechanical valve who suddenly develops severe cardiac failure. If the patient presents at a hospital where cardiac surgery facilities are not available immediate transfer should be arranged. 